Lexique vivant de la révolution et de la guerre en Syrie

\title{
Qualifier l'engagement des \\ Syriennes dans la \\ révolutionLes \\ retournements du mot \\ حرائر (femmes libres)
}

Emma Aubin-Boltanski ${ }^{1}$, Oussama Khalbous

${ }^{1}$ CéSor, Paris

Published on: Dec 29, 2021

License: Creative Commons Attribution-NonCommercial-NoDerivatives 4.0 International License (CC-BY-NC-ND 4.0). 
Cet article a été initialement publié sur le carnet de recherche du programme de recherche ANR Shakk (Projet-ANR-17-CE26-0021) le 24 juin 2020 : https://shakk.hypotheses.org $/ 2225$

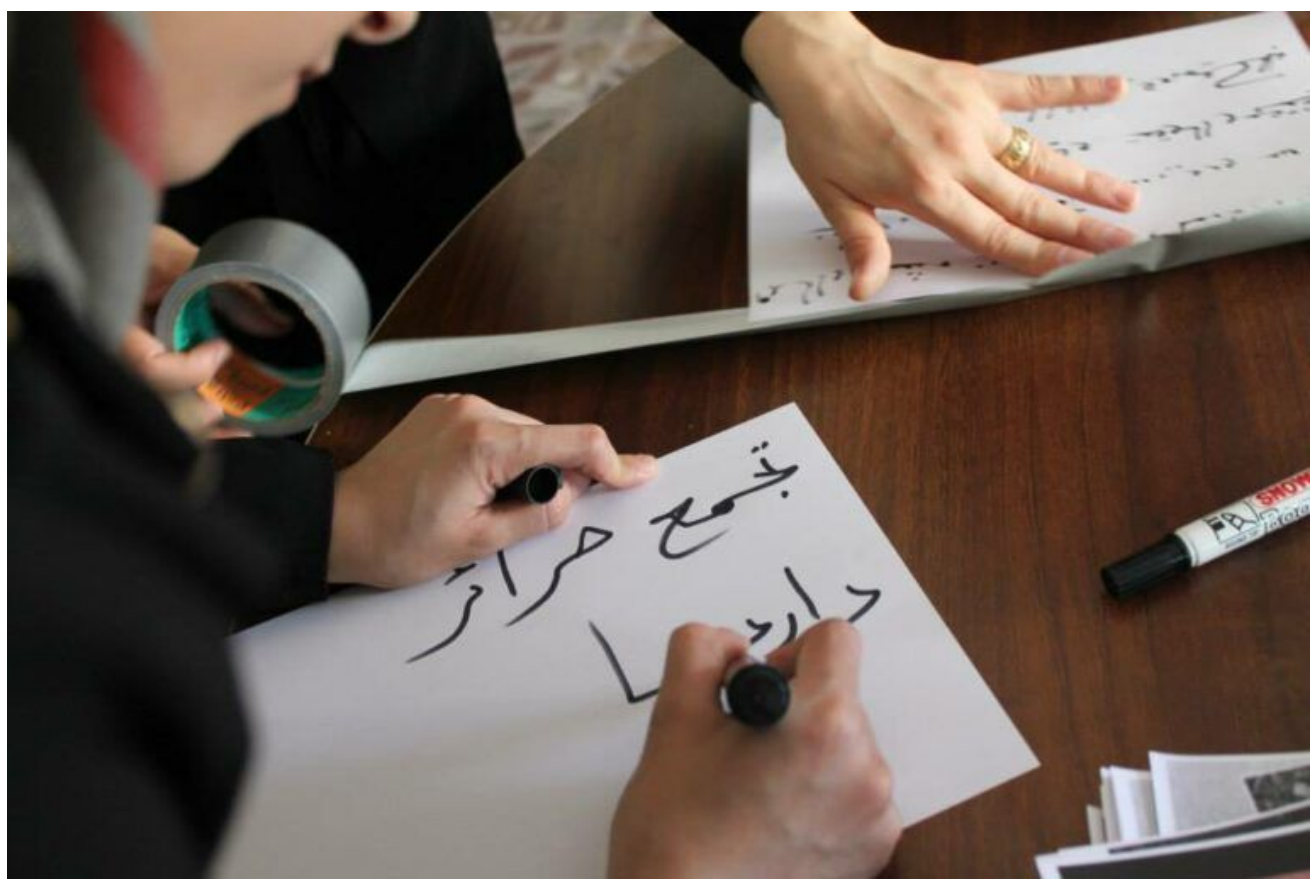

Une femme inscrit sur une feuille les termes "Rassemblement des femmes libres de Daraya”. Page Facebook Tajammu' harâ'ir dârayâ

https://www.facebook.com/freedoom.7ara2er/photos/ms.c.eJwzNT AwMjUxszQ2tjQ2MDDXMwXzLUzNzI3MjI1MAGPfBkI -.bps.a.50024 3753394101/500254693393007/?type=3\&theater

Voici neuf semaines que l'habitude est prise de voter pour un nom de vendredi de manifestation, quand le jeudi 12 mai 2011 le choix tombe : le lendemain sera baptisé « harâ'ir » (femmes libres). « Nous manifesterons pour la dignité de nos sœurs emprisonnées » annonce la page Facebook Révolution syrienne contre le régime de Bachar al-Assad . Ce vendredi des «femmes libres », les rassemblements sont immenses et c'est bien le mot « harâ'ir » qui est clamé et que l'on retrouve sur de nombreuses banderoles. Ce pluriel du terme « hurra » a une résonance singulière et archaïque. Il ne tardera pas à susciter l'ire des laïcs et une polémique au sein même de l'opposition. Dans ce camp, si certains le considèrent comme un mot «poétique ${ }^{1}$ » et neutre sans connotation religieuse, d'autres estiment que son usage généralisé est le signe d'une dérive islamiste et discriminatoire à l'égard des femmes. 
L'enquête dont nous restituons les grandes lignes dans cet article révèle des emplois variés, liés non seulement aux contextes d'énonciation - la valeur sémantique n'est pas la même en fonction des époques et des lieux-, mais aux positionnements politiques des personnes qui l'utilisent, parfois le brandissent tel un slogan. Notre propos n'est bien sûr pas de proposer la «véritable définition » du terme, mais d'en repérer quelques usages situés en Syrie à partir de 2011 et d'en saisir la charge à la fois sémantique et émotionnelle.

Essayons en premier lieu d'établir une brève chronologie de son apparition. Un sondage aléatoire sur la Toile effectué grâce à deux mots clés : « harâ'ir » et « thawra » (révolution) laisse penser que c'est d'abord au Yémen qu'il apparaît lors des manifestations de 2011. Dans ce contexte, il est en particulier utilisé par le Rassemblement yéménite pour la réforme (al-tajammu’ al-yamani li-l-islâh). Plus communément appelé al-Islâh, ce parti représente la branche yéménite des Frères musulmans. Pour désigner les femmes qui participent aux manifestations, ses membres déploient un large lexique à connotations religieuses qui comprend, en sus de « harâ'ir », les mots " sœurs » (shaqâ'iq), " glorieuses » mâjidât, « cristallines » (qawârir) et servantes de Dieu (amât allâh)르. Comme en Syrie, des femmes contestataires laïques se sont élevées contre ces appellations destinées à « confiner les femmes »ㅂ. À partir du Yémen, - les réseaux sociaux et les médias ont sans nul doute joué un rôle dans ce phénomène de contagion -, le terme connaît une diffusion dans l'ensemble du monde arabe en révolte, en particulier en Égypte et, de là, en Syrie. Dans un premier temps, le mot doit son succès aux Frères musulmans, mais très rapidement il se « libère » du lexique exclusivement frériste et de sa connotation islamiste pour être approprié par des centaines de milliers de manifestants qui vont en quelque sorte en proposer une nouvelle « version ».

Les références islamiques du terme n'épuisent pas ses significations, ni ses usages qui au fil des siècles ont évolué. Cependant, à l'époque actuelle, nombre de Syriens versés en sciences islamiques ou simplement lettrés, réduisent le mot à ses connotations religieuses. Quelles sont les références les plus fréquemment mobilisées pour le démontrer?

« Le titre harâ'ir à l'époque de Prophète et de ses compagnons implique pour celles qui le portent une série d'engagements : revêtir le voile, tenir son rang, ce qui signifie ne pas prendre part aux choses du quotidien et conserver sa chasteté, ce qui suppose de maintenir une stricte séparation des sexes dans la vie sociale » explique Mohammad Amir Nachir al-Ni'am, un islamologue syrien. Le mot n'apparaît pas dans 
le Coran, et est rarement employé dans le Hadith. En revanche, on le trouve dans les sources de la jurisprudence islamique, comme dans le Muwatta du théologien et traditionnaliste Malik ibn Anas (m. 796) :

« Malik m’a rapporté avoir entendu que ‘Umar b. Khattâb vit une esclave (amatân) appartenant à Abd Allâh b. 'Umar b. Khattâb. Elle avait revêtu la tenue des femmes libres. Il entra chez sa fille Hafsa et lui dit : n'ai-je pas vu une esclave (jâriya) circuler parmi les gens habillée comme une femme libre ? 'Umar désapprouvait cela »

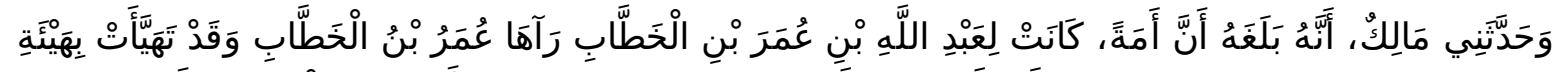

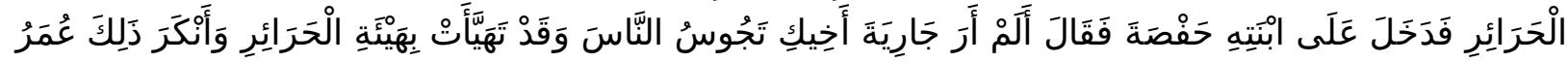

Ce « dit » indique que la notion harâ'ir et ses antonymes imâ' et jawâri $\underline{4}$ renvoient à des classes sociales. À l'époque du prophète, la société médinoise est divisée entre esclaves et sujets libres. Concernant les femmes deux catégories sociales sont opposées : d'une part, les harâ'ir ou «femmes libres » et, d'autre part, les imâ' et les jawâri de condition servile. À chacune, sont attachées des obligations vestimentaires : contrairement aux imâ' et aux jawâri qui n'ont pas le droit de se couvrir la tête, les harâ'ir ont le «privilège », en tant que «femmes bien nées » d'être voilées pour ne pas être apostrophées sur la voie publique $\underline{5}$.

« Celui qui souhaite rencontrer Dieu en état de pureté doit épouser des harâ'ir ».

$$
\text { مَنْ أَرَادَ أَنْ يَلْقَى اللَّهَ طَاهِرًا مُطَهَهرًا فَلْيََزََّْجِ الْحَرَائيَر }
$$

Dans ce hadith prophétique cité dans le chapitre sur le mariage du Kitâb al-sunan d'Ibn Mâja, une seconde idée tout à fait centrale apparaît, celle de « pureté ». Être une « hurra », c'est non seulement être « libre », mais c'est également être "chaste", « vertueuse », « honnête » et « de bonne famille ».

Dans les sources islamiques, harâ'ir renvoie donc à la fois à une classe sociale (femmes bien nées) et à des vertus (pureté, chasteté). Ces deux points sont importants pour comprendre la destinée singulière du terme à partir de 2011. Dans une société patriarcale et conservatrice comme la Syrie, les femmes qui font le choix de « sortir dans la rue » pour prendre part aux manifestations ou à des actions politiques sont souvent déconsidérées. Quand, de surcroît, elles ont le malheur d'être emprisonnées et donc probablement torturées et violées, la société les réduit à un état d'abjection. L'adoption du terme harâ'ir pour désigner ces femmes engagées serait-elle une façon d'en finir avec ces préjugés ? D’affirmer qu'elles demeurent « intactes » et « pures » 
malgré tout ? De garantir que les liens qui se nouent entre hommes et femmes dans l'arène de la révolution sont vertueux et honnêtes, en un mot désexualisés ?

Le terme fait l'objet d'un engouement exponentiel dans le camp de l'opposition, notamment sur les réseaux sociaux où de multiples groupes de « harâ'ir » apparaissent le plus souvent attachés à une localité précise : les harâ'ir de Daraya Qasyoun, de $\underline{\text { Mu'adhmiyya }}$, de $\underline{\text { Raqqaa }}$, etc. Ces collectifs sont engagés dans des actions sociales et d'aide à la population, notamment dans les régions « libérées » des forces du régime. Quelques-unes de ces femmes annoncent être prêtes à aller plus loin : prendre les armes et rejoindre les hommes au front « pour la révolution et notre patrie ». Sur les vidéos ou photos postées en nombre sur des pages Facebook ou des comptes YouTube, les « harâ'ir » sont strictement voilées et leurs visages dissimulés, probablement pour des raisons aussi bien religieuses que sécuritaires. La tonalité des appels prononcés par ces groupes est avant tout patriotique et citoyenne comme dans une vidéo mise en ligne par les harâ'ir de Damas le 22 juin 2011 : « Nous réclamons la liberté et refusons le confessionnalisme. Nous appelons à l'unité nationale » peut-on lire sur la pancarte centrale. Sur une autre « Non, non, non au pouvoir de l'appareil sécuritaire sur nos vies » ou encore : «Liberté pour un état civil et démocratique » (fig.1). En revanche, on ne note aucun appel à l'instauration d'un état islamique ou à un retour aux pratiques qui auraient été en vigueur dans la communauté musulmane à l'époque du Prophète Muhammad.

\section{Visit the web version of this article to view interactive content.}

Figure 1 : Une vidéo des harâ'ir de Damas postée sur Youtube le 22 juin 2011. (https://www.youtube.com/watch?v=gnONo7E5nv0)

Pourtant, au fil des semaines le label harâ'ir en vient à susciter crainte et désapprobation dans le camp de la révolution. Il est intéressant de noter l'évolution des émotions qu'il suscite chez une même personne. C'est, par exemple, ce qui transparaît dans Feux croisés, la chronique des quatre premiers mois de la révolution de la romancière Samar Yazbek. En exergue de son ouvrage, celle-ci rend hommage aux « mots » qui « l'ont aidée à affronter la peur ». Tel n'est pas le cas de harâ'ir qui finit par provoquer chez elle une vive inquiétude. Celle-ci se cristallise graduellement. À la date du 13 mai 2011, Samar Yazbek mentionne le choix du mot pour désigner le vendredi de manifestation sans paraître s'en alarmer. Un mois plus tard, le 26 juin 2011 , en revanche elle s'insurge contre ce «titre à la connotation islamique » que des femmes révolutionnaires de sa région (le littoral syrien) adoptent à leur tour pour leur 
page Facebook. J’aurais préféré « femmes [du Sahel]» précise-t-elle. Pour la romancière, il ne fait plus aucun doute que l'adoption de harâ'ir est significative d'une « dérive salafiste » de la révolution.

« Je ne comprends pas comment des opposants brandissent des banderoles sur lesquelles on peut lire 'harâ'ir de telle région'. Avec cette révolution nous voulons introduire de nouvelles valeurs ». Ces propos d'une jeune activiste de Damas ont été rapportés par le quotidien al-Akhbar le 8 mars 2012. À cette date, les Syriens sont censés, comme chaque année depuis 49 ans, célébrer « la révolution baasiste » de 1963. En 2012, les rebelles décident de « détourner » l'événement pour le transformer en « fête de la femme syrienne révolutionnaire » et ce en lien avec la journée internationale des droits des femmes. Ce jour-là, les manifestantes sont nombreuses à clamer haut et fort être des « révolutionnaires syriennes et pas des harâ'ir » (thâ'irât sûriyya. Lâ harâ'ir). Une page Facebook portant ce titre de « thâ'irât sûriyya. Lâ harâ'ir» a même été créée un mois plus tôt $\underline{6}$. Si ces personnes protestent contre le label harâ'ir, c'est qu'il leur paraît « antirévolutionnaire », enfermant les femmes dans leur statut traditionnel de « hurma », une expression qui désigne la « chose sacrée d'un homme », sa femme ou sa fille. Pour la jeune opposante interrogée par al-Akhbâr, l'emploi du mot harâ'ir signalerait l'influence des courants salafistes sur les mouvements révolutionnaires : «Nous faisons cette célébration de la femme syrienne pour ne pas devenir des harâ'ir comme certains le voudraient (...) Au fondement de notre révolution, nous réclamons la justice et rejetons toute forme d'exclusion. Je suis une révolutionnaire syrienne, je ne suis pas une hurma ».

Thâ'irât (femmes révolutionnaires) ${ }^{7}$ ou harâ'ir ? On peut observer de mars 2011 et ce jusque fin 2012 une forme d'hésitation entre les deux termes. C'est, par exemple, le cas dans deux vidéos qui en appellent à l'honneur des arabes pour porter secours aux femmes affligées de Syrie. La juxtaposition des deux mots dans le titre de la première est révélatrice de ce flottement : «ㄷi de dignité lancé du cour des thâ'irât harâ'ir »으․ Quant à la seconde, l'instabilité sémantique se retrouve dans le décalage entre son titre - « Thâ'irât sans frontière. Des cris lancés de la terre de Syrie » $\underline{9}$ - et cette phrase de présentation : " Vidéo transmettant les cris des femmes harâ'ir chastes de Syrie ». Ce phénomène est, à notre sens, significatif de l'ampleur de la rupture provoquée par le moment révolutionnaire : parce que tout ou presque est à (ré) inventer, une profusion de références et de symboles sont mobilisés entre lesquels les activistes tâtonnent, hésitent car leurs choix détermineront la nature du monde nouveau à construire. 
Dans une même séquence temporelle, le mot est employé par des groupes d'obédiences très différentes qui contribuent à élargir sa sémantique : si pour certains, harâ'ir en vient à devenir synonyme de «femmes révolutionnaires », à l'autre bout du spectre, d'autres emploient ce titre pour désigner des «femmes pures et innocentes », des « victimes à secourir ». Dans un même document ou discours ces significations se juxtaposent, voire se mélangent. Le titre est utilisé par des femmes probablement proches des frères musulmans comme ces mystérieuses « Harâ'ir de Damas dissidentes des qubaysiyyât » dont le discours dominé par une rhétorique islamiste antisoufie appelle à une « révolution contre une conception négative de la religion » et rappelle avec insistance les rôles qui incombent aux femmes sur terre. Il est aussi brandi par des salafistes. Ces deux groupes ne paraissent pas avoir un usage similaire du terme. Deux chansons que tout oppose permettent d'en repérer les emplois différenciés : " Harâ'ir al-shâm. Yâ wâqi' al-dhull » (Harâ'ir al-Shâm. Ô réalité de l'humiliation) un hymne à la révolution syrienne aux accents résolument salafistes et « Mahadash yeksernî » (Personne ne me cassera), un clip d'un groupe égyptien appelé La terrasse des harâ'ir de la révolution. Dans la première, un homme vilipende a cappella « l'ignominie des Arabes » accusés de ne pas porter secours aux " harâ'ir du Shâm », des femmes sans défenses, désespérées et déshonorées par des mercenaires. Le second chant est au contraire scandé par une polyphonie de voix féminines avec un accompagnement musical très rythmé. Sur les images fixes qui se succèdent dans le clip, on voit des manifestantes égyptiennes et syriennes faisant le « R4BIA », le signe de ralliement des partisans des Frères musulmans qui consiste à dresser quatre doigts d'une main en repliant le pouce (fig. 2), ou encore lancer des projectiles $\underline{10}$. "Je suis une fille, mais je vaux 100 hommes. La liberté n'est pas facile. Mais l'oppresseur décampera demain. Je suis une fille, mais personne ne peut me casser. Je suis une patriote, fille de mon pays » sont les paroles qui reviennent en boucle. Contrairement au premier document sonore, ce chant met en avant la capacité d'agir et de combattre des «femmes libres » dans l'arène de la révolution. 

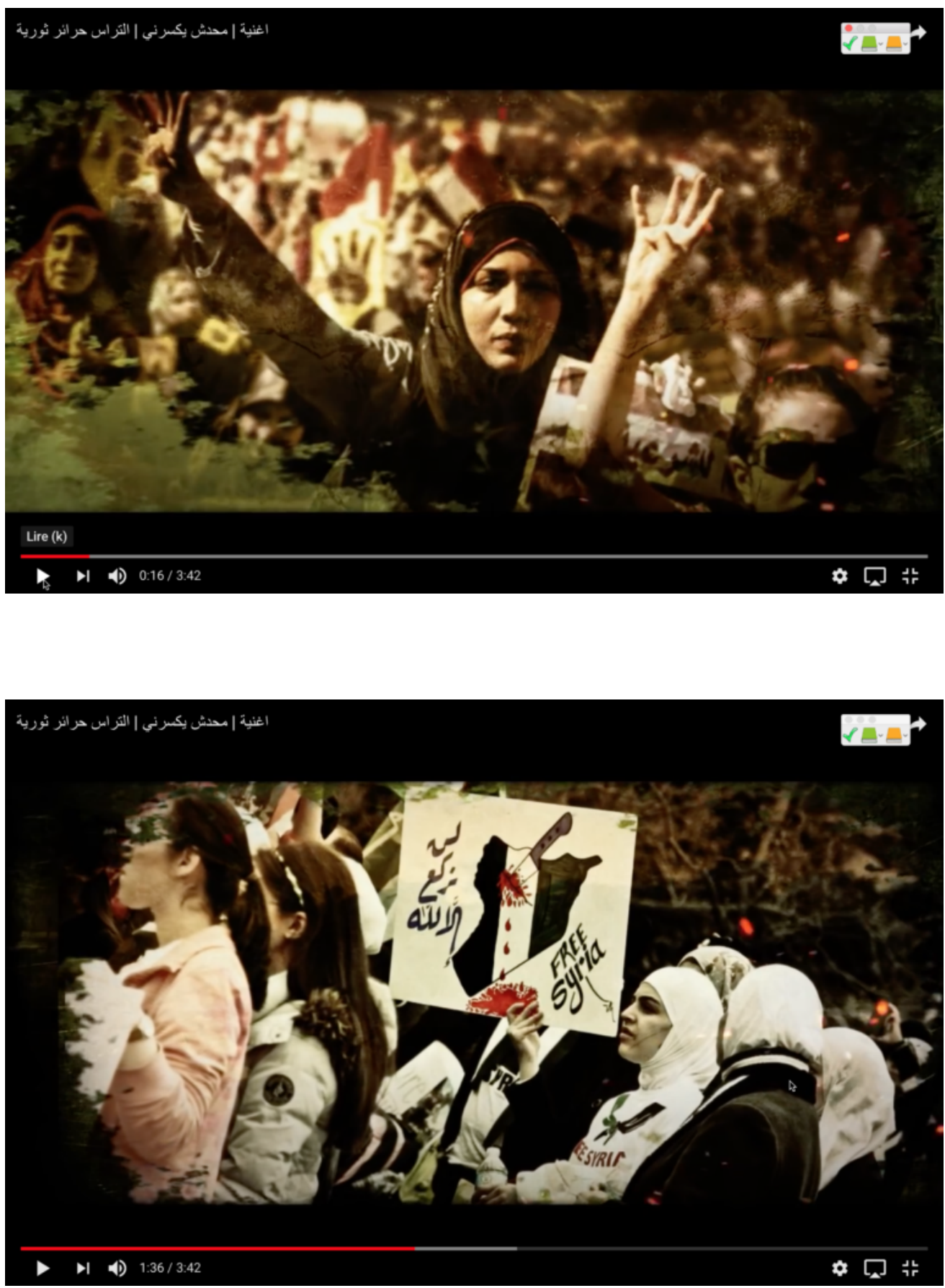

Figures 2 : captures d'écran du clip "Mahadash yekserni”. Sur la première, deux femmes font le R4BIA; sur la seconde, une manifestante brandit une pancarte sur laquelle on peut lire en arabe: "Nous ne nous prosternons que devant Dieu" (https://www.youtube.com/watch?v=92g9nPvhGnk) 
Surtout, simultanément, le mot harâ'ir est adopté par des groupes résolument laïcs, comme « Douma City » un collectif de nassériens qui le 5 avril 2012 appelle les femmes de Douma à manifester sur un encart placardé un peu partout dans la ville : " Aux harâ'ir de Douma. Nous vous appelons à manifester jeudi 5 avril 2012 à quatre heures devant l'école secondaire des filles de Douma (Ghâzi) pour les martyrs de la liberté. Vive la Syrie libre éternelle qui n'accepte pas l'injustice et à bas Bachar alAssad. Si Dieu veut, la victoire sera proche grâce à notre glorieuse révolution et notre ténacité. À nous la victoire et à la bande d’Assad la damnation » (fig. 3). 


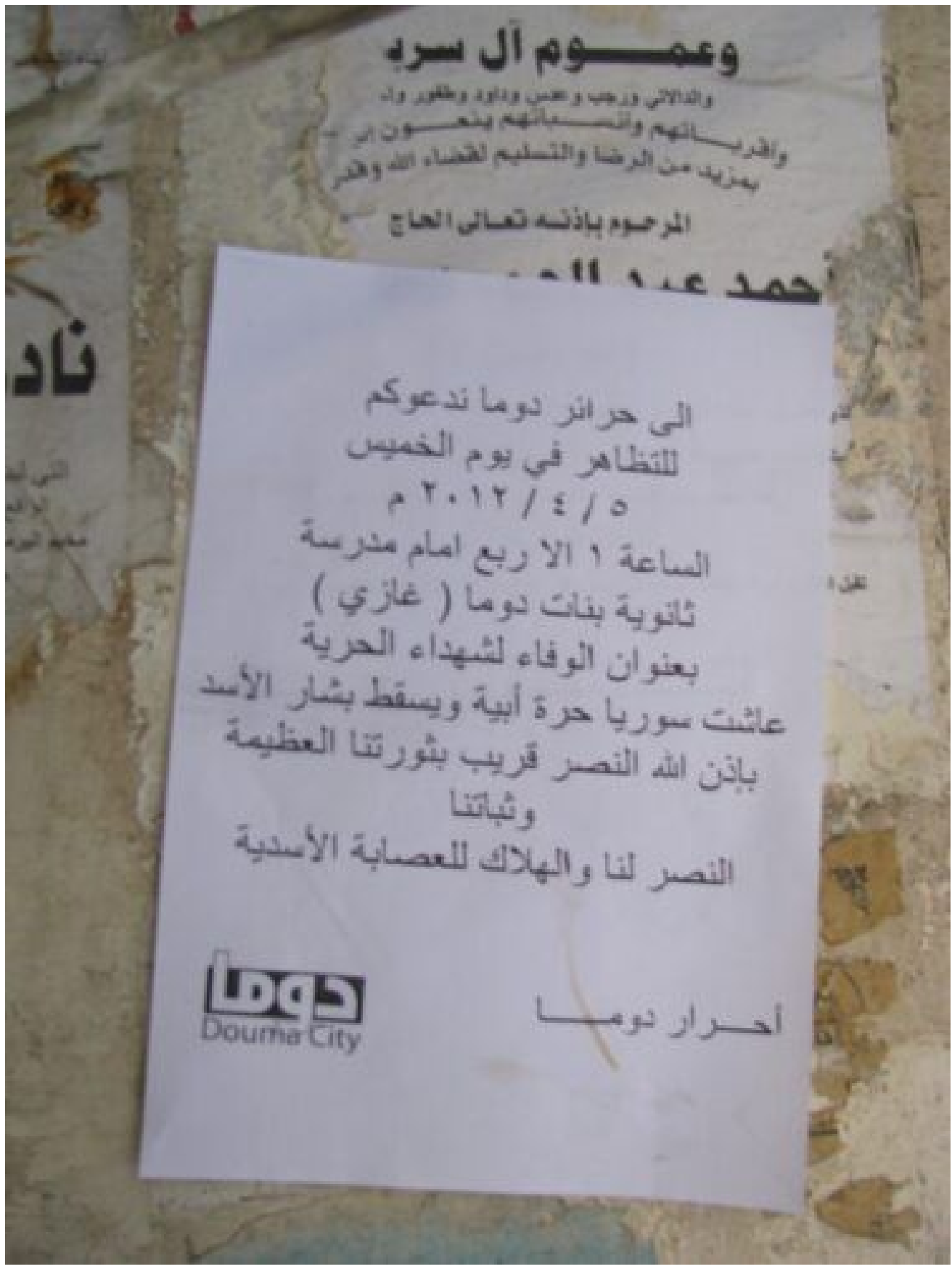

Figure 3: Appel à manifester du collectif Douma city. Photo : anonyme

Figure 3 : Appel à manifester du collectif Douma city. Photo : anonyme

De la même façon, dans le célèbre chant « Irhâl yâ Bachâr » (tire-toi Bachar) d’Ibrahim Kachouch, le terme qui apparaît à la dernière strophe est tout à fait délesté de sa charge religieuse. «Saluez les harâ'ir de Hama et tous les habitants de Hama, La Syrie veut sa liberté ! La Syrie veut sa liberté ! » reprennent en cœur des foules immenses 
de manifestants réunis au centre de Hama en juin 2011. Deux vers plus haut, Ibrahim Kachouch tourne en dérision les discours qui réduisent les révolutionnaires à des « terroristes » et des « salafistes »: «Quand nous avons revendiqué la liberté, on nous a appelés terroristes, La Syrie veut la liberté ! Quand nous avons exigé nos droits, On nous a appelés salafistes ». Autre chant, autre lieu : en novembre 2011, à Homs des milliers de protestataires, que des hommes entonnent « Thawra thawra » (révolution, révolution). En rang et serrés épaule contre épaule, ils hurlent ensemble « Révolution, révolution. Syrie, Syrie. Nous refusons le confessionnalisme... Nos femmes sont des harâ'ir, les descendantes d'al-Khansâ' et de Khawla». Ici un lien est établi entre les harâ'ir et deux figures féminines du $7^{\mathrm{e}}$ siècle devenues l'une et l'autre des emblèmes du soulèvement de 2011. Al-Khansâ' (poétesse des $6^{\mathrm{e}} / 7^{\mathrm{e}}$ siècles, contemporaine du Prophète) symbolise la sœur éplorée : elle doit sa célébrité à ses élégies funèbres prononcées à la mémoire de ses frères tués au combat. Khawla bint al-Azwar quant à elle, est l'icône de la guerrière : Elle combattit dans les rangs de l'armée musulmane lors des conquêtes arabes.

Harâ'ir est donc entré dans le lexique de la révolution syrienne en 2011. Même s'il fait l'objet d'une polémique, il finit par s'imposer dans différents cercles de l'opposition, religieux comme laïcs. Signe de son hégémonie, la propagande du régime de Damas elle-même en vient à se l'approprier. Avant 2011, le terme était absent du vocabulaire officiel. Seule exception : nombre de Syriens ont encore en tête un chant patriotique composé au lendemain de la guerre de 1973 et interprété par Mohammad Salman, Najah Salam et Mohammad Jamal, appelé Sûriyya habîbatî (Syrie, mon amour). À la deuxième minute, Najah Salam accompagnée d'un roulement de tambour et de violons compare la Syrie à une « qal'at al-ahrâr wa-l-harâ'ir » (une forteresse d'hommes et de femmes libres). Le jeu de captation et de subversion des mots de la révolution par le pouvoir concerne plusieurs termes, notamment ceux de « dignité » (karâma) et de « justice » ('adâla) que l'on retrouve jusque dans les discours de Bachar et Asma alAssad. Comment s'est-il opéré dans le cas qui nous intéresse ? Harâ'ir a été choisi comme le titre d'un feuilleton du mois de Ramadan produit en 2015 par Syriana, une société de production proche du régime. Le scénario se déroule pendant la Première Guerre mondiale et s'achève au moment où le Mandat français commence. Il est centré autour de plusieurs personnages féminins fictifs et réels - dont Marie Ajami, la célèbre féministe syrienne qui fonda dans les années 1920 un club littéraire à Damas et Nazik Abid une autre féministe connue pour son opposition aux Français et notamment pour avoir dirigé une unité d'infirmières de l'armée arabe lors de la bataille de Maysaloun $\underline{11}$. Le feuilleton retrace le destin de ces «femmes libres » en 
trente épisodes. Comme de nombreuses séries télévisées qui l'ont précédé, il adopte une rhétorique féministe critique à l'égard de la société patriarcale syrienne $\underline{12}$. C'est en particulier un milieu traditionnel sunnite largement fantasmé qui est visé à travers le personnage d'un homme - un marchand de Damas - qui n’hésite pas à spolier sa belle-sœur et à forcer une jeune orpheline à l'épouser ou encore un « pacha » vivant dans un palais où sont recluses ses « hurma » et ses « jawâri » (femmes esclaves). Les héroïnes se soulèvent contre ces injustices et leur combat finit par se confondre avec la lutte nationaliste contre le Mandat français. Au 29e épisode, elles manifestent contre les Français et pour « une Syrie libre ». Nazik Abid s'exclame : « Nous avons un régime, une loi et une armée pour nous protéger. Nous n'avons pas besoin du Mandat français pour le faire. » «Le temps de la peur est parti » crie Marie Ajami comme en écho à un chant de la révolution intitulé « Les murs de la peur sont tombés ». Les références aux évènements que vit la Syrie depuis 2011 émaillent la narration : dans cette scène, au moyen d'une distorsion historique (en 1920, s'il existe un « régime » c'est celui de l'éphémère Royaume arabe syrien avec lequel Nazik Abid était liée, ce que le réalisateur Bassel al-Khatib refuse de mentionner dans son film sous prétexte que le roi Fayçal aurait « donné la Palestine aux juifs » $\underline{13}$ ). Par un effet de contraction temporelle, l'impression est donnée que ces femmes libres appuient « déjà » (du lieu où elles se situent, Damas en 1920) le régime de Bachar al-Assad et reprennent à leur compte la rhétorique « anti-occidentale » de celui-ci. C'est probablement au personnage de Bassima, qui dans la série représente « la femme ordinaire » syrienne, que revient la fonction d'activer le principe orwellien de « mutabilité du passé » qui consiste ici en une réécriture de l'histoire présente par le truchement du passé $\underline{14}$. Bassima est jouée par Sulaf Fawakharji, une star du petit et du grand écran dont les positions pro régimes sont connues de tous les Syriens. Dans la scène de la manifestation contre les autorités mandataires, le réalisateur Bassel al-Khatib a choisi d'insérer plusieurs gros plans de son visage larmoyant alors qu'elle reprend ce slogan que des centaines de milliers de Syriens ont clamé à partir de mars 2011 : « sûriya hurra » (Syrie libre). Le cadrage rapproché rend la scène intemporelle. L'effet est saisissant : qui crie ? Bassima ou Soulaf Fawakharji ? Sommes-nous en 1920 ou en 2015 ? Quel est l'objectif de cette subversion du mot hurra, si ce n'est de l'altérer pour mieux assujettir ? (fig. 4) 


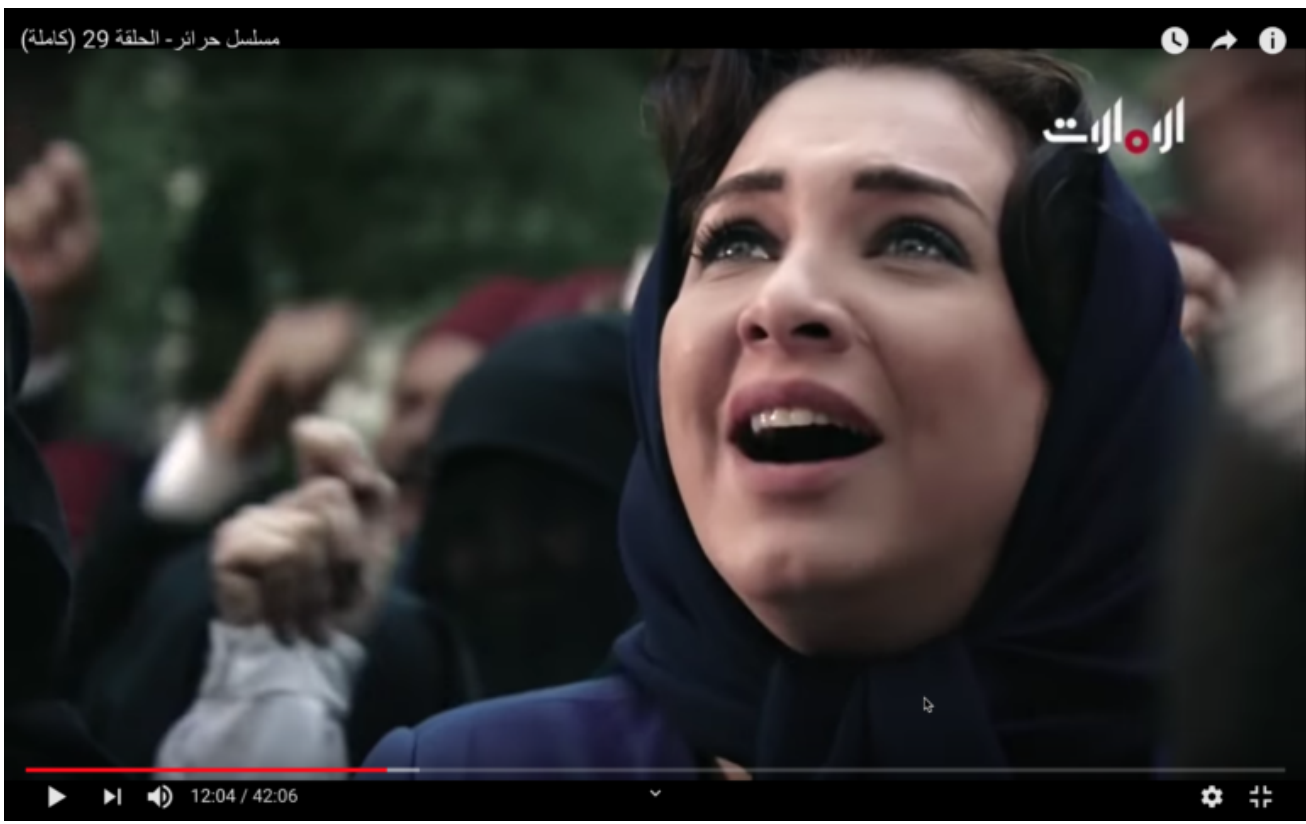

Figure 4. Capture d'écran du 29e épisode de Harâ'ir. Le personnage de Bassima joué par Soulaf Fawakharji entonne le slogan "Syrie libre" (https://www.youtube.com/watch? $\underline{v=890 t H A H t y b M}$ )

Ajoutons que dans cette série, la mise en scène des corps des « harâ'ir » révèle aussi le point de vue du réalisateur sur les évènements politiques en cours dans son pays: cheveux longs tombant en cascade jusqu'à la taille ou, au contraire, coupés au carré pour Marie Ajami et Nazik Abid archétypes de «femmes modernes », tenues avenantes, visages savamment maquillés, fréquents regards caméra interpellant directement le téléspectateur, etc. Le contraste avec les vidéos postées par les « femmes libres » de la révolution est saisissant : les visages y sont généralement dissimulés et les regards absents, les cheveux sont voilés et les tenues d'une sobriété extrême.

Pour finir, quelques remarques sur les péripéties du mot dans ses traductions en anglais et en français.

Dans un article paru dans la revue en ligne al-Jumhuriya en 2019 sur la violence de la traduction, Eylaf Bader Eddin se penche sur la traduction de harâ'ir en anglais. Il critique avec une grande virulence l'option choisie par Max Weiss pour rendre le sens du mot dans un passage de l'ouvrage Feux croisés, paru en anglais en 2012. Comme nous l'avons déjà mentionné, Samar Yazbek fait allusion dans sa chronique à la création d'un groupe de « harâ'ir du littoral syrien » et s'insurge contre le choix de ce label. Pour traduire harâ'ir et probablement rendre compréhensible la colère de la 
narratrice à un lecteur anglophone, Max Weiss opte pour la formule « free virgin women » et renvoie à une note de bas de page pour expliquer dans une perspective très orientaliste la connotation islamique du mot. Pour Eylaf Bader Eddin en procédant ainsi, le traducteur « islamise » le terme avant les Syriens eux-mêmes, car en juin, précise-t-il les manifestations en Syrie n’avaient aucun caractère islamiste. La critique cible l'ajout de « virgin » qui connote négativement le mot et elle vilipende la note de bas de page qui non seulement propose une définition entièrement rabattue sur les sources religieuses (Coran, hadith et fiqh), mais identifie tous les groupes de harâ'ir apparus en 2011-2012, à des formations « plus ou moins affiliées aux Frères musulmans » $\underline{15}$. La charge n'est pas sans fondement, mais elle paraît excessive. L'objectif est de démontrer qu'à travers cette traduction une représentation orientaliste et négative de la révolution syrienne se dessinerait. Cependant, Eylaf Bader Eddin ne précise pas que c'est Samar Yazbek elle-même qui, en juin 2011, considère que le terme a une connotation islamiste et qu'à une autre occurrence, lorsqu'il est question du « vendredi des harâ'ir », Max Weiss opte pour une traduction bien plus neutre « Friday of Free Women ».

Rania Samara, la traductrice de ce texte en français a, quant à elle fait le choix de traduire le terme harâ'ir de deux façons différentes. Si à la première occurrence elle choisit simplement « vendredi des femmes libres », à la seconde plus problématique, elle opte pour «dames » convoquant l'emploi ancien du terme, synonyme de femme bien née, femme de condition, femme de qualité, noble. Le choix est intéressant, mais ne permet pas de comprendre les raisons de la colère de la narratrice ni en quoi ce « titre » en français de « Dames du littoral syrien » aurait cette « connotation islamique » que lui reproche l'autrice.

Un second exemple doit être mentionné : il s'agit de l'ouvrage Q Qissât makân,_quisât

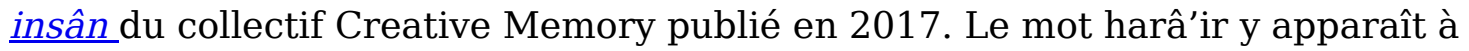
plusieurs reprises pour désigner ces groupes de femmes qui se forment à partir de juin 2011 dans l'objectif de soutenir la révolution en s'engageant dans des actions sociales et caritatives dans leurs localités respectives (notamment Daraya, mais également Damas, Douma et Nabk). Dans cet ensemble de chroniques locales des évènements de 2011 à 2015, il n'est jamais fait allusion au débat suscité par le terme. Dans la

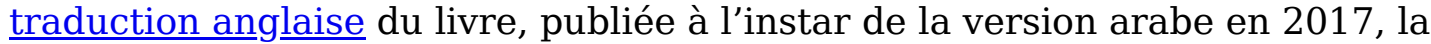
traductrice Rana Mitri opte pour la locution « Free Women ». En revanche, deux ans plus tard, Nathalie Bontemps la traductrice du texte en français, choisit l'expression « citoyennes libres » qui permet de signifier l'engagement social et politique de ces collectifs féminins. 


\section{Bibliographie}

La bibliographie de cet article est disponible dans le groupe Zotero du Lexique (https://www.zotero.org/groups/4550572/syria_lexicon/collections/7Z7CQD73).

\section{Footnotes}

1.

Une recherche approfondie sur l'emploi du terme dans la poésie contemporaine doit être menée. On le retrouve dans l'un des vers du célèbre poème Samba de Nizzar Kabbani :

. Consulté le 11 janvier 2022.

https://web.archive.org/web/20220111155532/https://nizarq.com/ar/poem186.html.. 2.

Sur ce sujet cf. Arwa Abdo Othman, « Lecture du printemps féministe au Yémen », Bidâyât, été 2013 https://www.bidayatmag.com/node/401, traduit en français par Nathalie Bontemps dans Yémen. Écrire la guerre (Classique Garnier, 2018. Pour chacun de ces termes, la traductrice précise le hadith qui en constitue la référence.

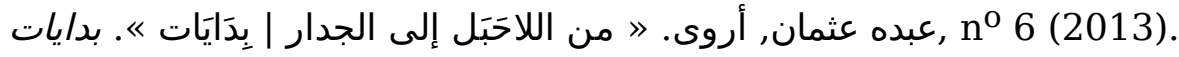
https://web.archive.org/web/20200927071309/https://bidayatmag.com/node/401.

Abduh Othman, Arwa. « Lecture du printemps féministe au Yémen », 29-42. Classiques Garnier, 2018. https://doi.org/10.15122/isbn.978-2-406-08365-8.p.0029.. 3. Ibid. $\triangleq$

4. Sur la différence entre ama (pl. imâ') et jâriya (pl. jawâri) cf. Myrne, Pernilla. $A$ Jariya's Prospects in Abbasid Baghdad. Oxford University Press, 2017. https://doi.org/10.1093/oso/9780190622183.003.0004. in Gordon, Matthew S., et Kathryn A. Hain, éd. Concubines and Courtesans. Vol. 1. Oxford University Press, 2017. https://doi.org/10.1093/oso/9780190622183.001.0001. p. 52-74. 5. Chaumont, Eric. « La notion de 'awra selon Abû l-Hasan 'Alî b. Muhammad b. alQațân al-Fâsî (m. 628 /1231) ». Revue des mondes musulmans et de la Méditerranée, $\mathrm{n}^{0}$ 113-114 (7 novembre 2006): 109-23. https://doi.org/10.4000/remmm.4052.. 
6. Cette page Facebook a été aujourd'hui supprimée, il n'en reste plus que l'URL : https://www.facebook.com/pages/371677209525304/ثائرات-سوريا-لاحرائري. Elle est citée dans l'article d'Al-Akhbar sus-mentionné. $\doteq$

7. Ce terme fera l'objet d'un article séparée. $\triangleq$

8. Sakhrat karâma min qulûb thâ'irât harâ'ir : https://www.youtube.com/watch?

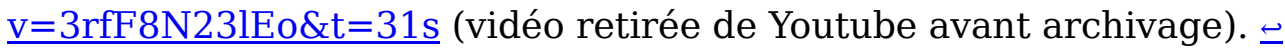

9.

Thâ'irât bilâ hudûd. Sakhrât min ard al-Shâm :

revolutionistwithout. ثائرات بلا حدود (1) : صرخات من أرض الشام, 2011. https://www.youtube.com/watch?v=PVqDSTNaDdk.

10. Rabi'a stylisé R4BIA signifie $4^{\mathrm{e}}$ en arabe. A l'origine, il s'agit d'une référence à la place Rabi’a adawiyya où un sit-in anti Général Sissi a été réprimé dans le sang_ par le pouvoir en août 2013.

11. Thompson, Elizabeth. « Le mouvement féminin et l'essor de l'État-providence colonial en Syrie (1920-1946) ». Clio, no 33 (1 mai 2011): 107-24.

https://doi.org/10.4000/clio.10030.

12. Sur ce point, cf. Joubin, Rebecca. The politics of love: sexuality, gender, and marriage in Syrian television drama. Lanham: Lexington Books, 2013. http://www.worldcat.org/oclc/1227504090.. En revanche, contrairement aux séries télévisées analysées dans cet ouvrage, dans harâ'ir, la mise en accusation de la société patriarcale ne nous semble pas constituer une critique détournée du régime des Assad. $\_$

13.

Sur ce point cf. un article paru sur la querelle entre la scénariste et le réalisateur au sujet du refus de ce dernier de conférer une place positive au Roi Fayçal dans la série et ce au prix d'une distorsion. Amîna Melhem, "Kâtibat harâ'ir tuthîr jadalan », al-Bînâ' :

ملحم, آمنة. 》 كاتبة 》حرائر《 ثثير جدلاً حول نهاية العمل باسل الخطيب لـالبناء《: لم نأتِ بأيّ إضافة إلّا ل Al-binaa Newspaper. Consulté le 11 janvier 2022. https://web.archive.org/web/20170816052814/https://www.al$\underline{\text { binaa.com/archives/article/58650. }}$. 
14. Sur ce principe récurrent dans le cinéma et dans les séries, cf. Boëx, Cécile. Cinéma et politique en Syrie: écritures cinématographiques de la contestation en régime autoritaire: 1970-2010. Collection L'ouverture philosophique. Série arts vivants. Paris: L’Harmattan, 2014. http://www.worldcat.org/oclc/903317526.. 15. A l'appui de la critique d'Eylaf Bader Eddin, citons le dictionnaire de Blachère, Régis, Moustafa Chouémi, et Claude Denizeau. Dictionnaire arabe-français-anglais: langue classique et moderne = Arabic/French /English dictionary. Paris: G.-P. Maisonneuve et Larose, 1967. http://www.worldcat.org/oclc/726192885., tome IV, fasc. 38, Paris, Maisonneuve \& Larose, 1978, p. 2392-2400. On y voit bien que hurr(a) renvoie surtout à la condition d'homme/femme libre / de bonne famille / de haut lignage / de race pure / d'honneur, etc. ; pour les femmes, aussi : vertueuse, chaste, pure (ce qui ne veut pas dire vierge). Il n'y a qu'une expression où on réfère clairement à la virginité : layla ḥurra nuit pure, chaste (nuit nuptiale passée [par la mariée] sans relation sexuelle, dans la chasteté, sans perdre sa virginité. $\subseteq$ 